\title{
Genotyping of Lipoprotein(a) Gene Variants in Coronary Artery Disease in Indian Population
}

\author{
Anu RI ${ }^{1}$, Charanjeet Kaur ${ }^{2}$, Prabhash Bhavsar ${ }^{3}$, Jagdish Prasad ${ }^{4}$
}

\begin{abstract}
India is undergoing an epidemiological transition and an alarming rise in the prevalence of coronary artery disease (CAD). The etiopathology of the disease is evolving to a more complex setting with the discovery of novel risk factors. In the quest for non-traditional risk factors, we found that Lipoprotein(a) gene and its product are unique and pertinent to the Indian population. To unravel the genetic blueprint of the variations within the LPA gene in Indians, we studied novel exonic and intronic SNPs in 60 patients with angiographically proven CAD and matched them with healthy volunteers. From the study, we observed that in our patients, LPA aspirin genotype rs3798220 did not cause variation in Lp(a) levels in either of the two groups which is a novel finding as it is at odds with most published studies from other populations, on this variant. The polymorphic allele of SNP rs1321196 increased serum Lp(a) levels but the presence of the variant was however not limited to the patient population. The SNP rs9364564 did not influence Lp(a) levels in either of the two groups of the study. The study is unique as it bears witness to the novelty of the genotype background in Indian patients with CAD.

Keywords: Allele-specific PCR, Cardiovascular genetics, Coronary artery disease, Lipoprotein(a), LPA aspirin genotype, LPA gene polymorphism, Molecular cardiology, rs1321196, rs3798220, rs9364564.

Indian Journal of Medical Biochemistry (2021): 10.5005/jp-journals-10054-0178
\end{abstract}

\section{INTRODUCTION}

The prevalence of coronary artery disease (CAD) is reaching epidemic proportions in India with a fourfold rise in the past 40 years. ${ }^{1}$ The estimated prevalence of cardiovascular disease in India is 54.5 million, as of $2016 .{ }^{2,3}$ Traditional risk factors for CAD do not provide an adequate explanation for the epidemic proportions of the disease. The non-traditional risk factors have proved to bridge this gap to an extent, together with the fact that CAD being highly heritable, bringing to light the complex polygenic backdrop of the setting of the disease.

A multi-centered case-control study, PROCARDIS (Precocious Coronary Artery Disease) investigated 3,145 cases and 3,352 control subjects to evaluate 2,100 candidate genes using evaluation of single-nucleotide polymorphisms (SNPs). The study found three genomic loci associated with CAD viz. 6q26-27, 9p 21, and 1p13. Of these, the locus 6q26-27 encompassing the LPA gene that encodes for Lipoprotein(a) had the strongest association with CAD. ${ }^{4,5}$

Numerous trials have shown us that patients with $\operatorname{Lp}(a)$ levels above $30 \mathrm{mg} / \mathrm{dL}$ had markedly increased risk of CAD and susceptibility to occlusive complications after various interventions. ${ }^{6}$ A high level of Lipoprotein(a) is an important determinant of CAD and is projected to be one of the strongest biomarkers of premature heart disease. ${ }^{7}$ It is noteworthy that $\mathrm{Lp}(\mathrm{a})$ has been proved to be an independent risk factor of CAD by the Framingham Heart Study and Premature CAD attributable to high Lp(a) was double that of hypertension or diabetes mellitus as concluded by the Copenhagen City Heart Study.

Structurally, $\operatorname{Lp}(\mathrm{a})$ is a plasma lipoprotein consisting of a cholesterol-rich LDL-like particle, having apolipoprotein(a) attached to apo $B$ via a disulfide bond. ${ }^{8}$ The basic LDL component has a lipid core formed by cholesteryl esters and triglycerides, which is surrounded by a layer of unesterified cholesterol, phospholipids, and an apolipoprotein B-100 (apo B-100) molecule. The apo "a" particle surrounds this complex and is attached by a disulfide bond to the apo B-100 component as shown in
${ }^{1}$ Department of Clinical Biochemistry, MVR Cancer Center and Research Institute, Calicut, Kerala, India

${ }^{2}$ Department of Biochemistry, Vardhman Mahavir Medical College and Safdarjung Hospital, New Delhi, India

${ }^{3}$ Department of Biochemistry, Government Medical College and Shri Haridev Joshi General Hospital, Dungarpur, Rajasthan, India

${ }^{4}$ Department of Cardiothoracic and Vascular Surgery, Vardhman Mahavir Medical College and Safdarjung Hospital, New Delhi, India

Corresponding Author: Anu RI, Department of Clinical Biochemistry, MVR Cancer Center and Research Institute, Calicut, Kerala, India, Phone: +91 9971272384, e-mail: anuevangelineiris@gmail.com

How to cite this article: RI Anu, Kaur C, Bhavsar P, et al. Genotyping of Lipoprotein (a) Gene Variants in Coronary Artery Disease in Indian Population. Indian J Med Biochem 2021;25(2):76-82.

Source of support: Indian Council of Medical Research (ICMR) under grant for MD/MS/MCh/DM Thesis (2015)

Conflict of interest: Anu R I is the Project Head of FM Oncology Breast cancer patient portal services, by Future Medicine Co.

Figure 1. The apo(a) moiety is encoded by the LPA gene located on chromosome $6 q$ 26-27, consists of multiple repeated segments or domains called Kringles. The Kringles are tri-looped structural units containing about 77-79 amino acids. They are connected by linker regions of 26-36 amino acids. ${ }^{9}$ Apo "a" has Kringles numbered IV and V, and a protease domain that is catalytically inactive. ${ }^{10}$ Kringle IV types I and III to 10 exist only as single copies, whereas Kringle IV type II is present in multiple copies in an individual. This special variation in the number of copies from 3 to more than 40 copies, is responsible for the apo "a" size and molecular weight heterogeneity in a person ${ }^{11}$ with a range between 300 and $800 \mathrm{kDa} .{ }^{10,12}$ The isoform size of the apo "a", which thus depends on the number of repeats of the KIV type II repeats, has high inter-individual variation. Each Kringle has

() The Author(s). 2021 Open Access This article is distributed under the terms of the Creative Commons Attribution 4.0 International License (https://creativecommons. org/licenses/by-nc/4.0/), which permits unrestricted use, distribution, and non-commercial reproduction in any medium, provided you give appropriate credit to the original author(s) and the source, provide a link to the Creative Commons license, and indicate if changes were made. The Creative Commons Public Domain Dedication waiver (http://creativecommons.org/publicdomain/zero/1.0/) applies to the data made available in this article, unless otherwise stated. 


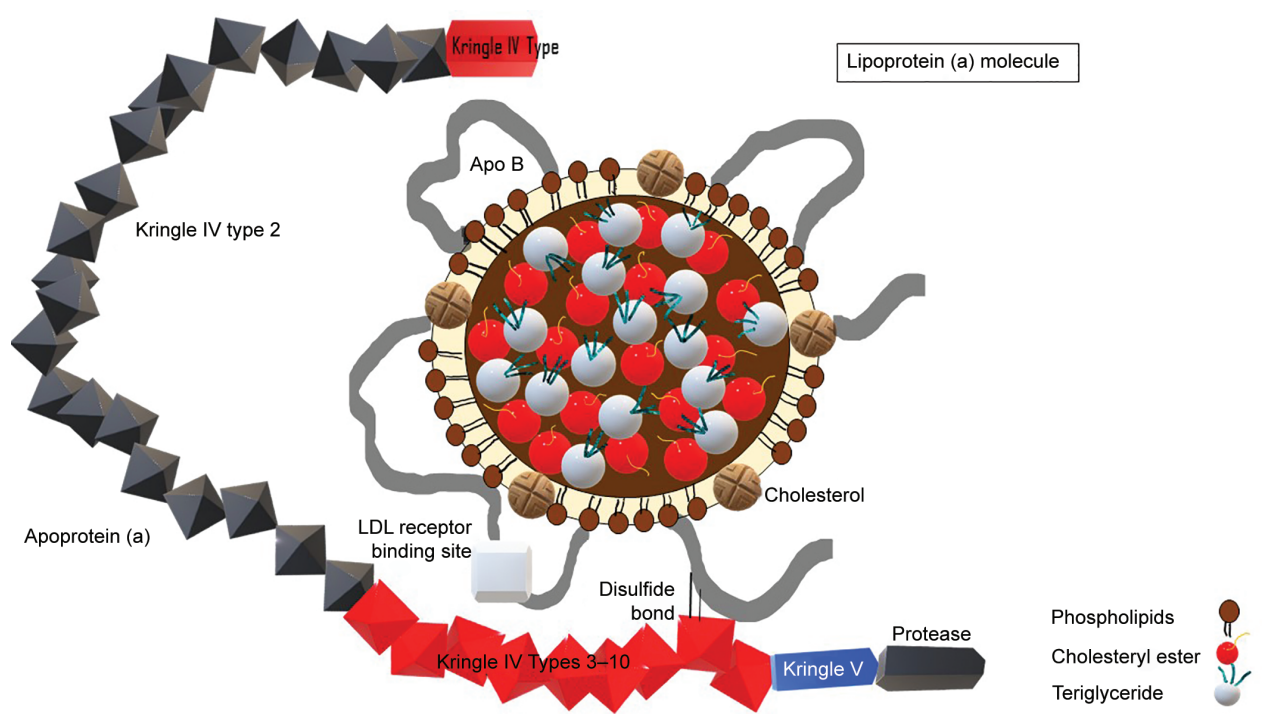

Fig. 1: Graphical structure of lipoprotein (a) molecule

three disulfide bonds and a peculiar folding pattern that serves as binding motifs ${ }^{13}$ probably for lysine or lysine analogs. ${ }^{14}$ Serum $\mathrm{Lp}(\mathrm{a})$ levels display high inter-individual variations and may range from $<1$ to $>1,000 \mathrm{mg} / \mathrm{dL}$, with a possibility of being absent totally from the blood. ${ }^{15} \mathrm{Lp}$ (a) concentrations and their relationship with the cardiovascular disease vary across races or ethnicities. ${ }^{16} L P A$ gene polymorphism is responsible for this wide variation in the serum Lp(a) levels with a heritability of $73-90 \%{ }^{17,18}$

Despite a few studies proving the association between risk of development of CAD and increased Lipoprotein(a) levels, there is a paucity of literature revealing the existence or association of the LPA gene polymorphism and risk of development of atherosclerotic cardiovascular diseases (ASCVD) in Indian population. Hence, this study aims in bringing to light the SNPs in the LPA gene which may prove to be significant in the Indian population. This will further help us in further research in the arena of treatment and prevention of patients with CAD.

\section{Materials and Methods}

The study was conducted on 60 patients with angiographically proven CAD, admitted for coronary artery bypass graft (CABG) and the age group varied from 45 to 70 years. Fifty apparently healthy individuals were selected as a control group. Patients suffering from hepatic, renal, or thyroid dysfunction and patients taking drugs such as niacin were excluded from the study. After obtaining informed consent, venous blood was collected in two vacutainer collection tubes. The blood from the EDTA tube was used to extract genomic DNA and that from the "no additive" tube was used for testing Lipoprotein(a) using Immunoturbidimetry, and routine parameters. Ethical approval was obtained from Institution Ethics Committee before the commencement of the study. Statistical analysis was performed using SPSS 22.0 software. Data are presented as mean (standard deviation) unless otherwise specified. The difference in the mean baseline values of various parameters was analyzed by unpaired $t$-test or Mann-Whitney test depending upon whether the values were parametric or non-parametric. Nominal variables were analyzed using $\chi^{2}$ and Fisher's exact tests. Correlation between two parameters was made using Pearson's correlation. A $p$ value of $<0.05$ was considered significant. The risk of development of CAD was evaluated by odds ratio calculation.

\section{Molecular Genetics Experiment}

Extraction of DNA was performed using QIAamp Blood Mini Kit from Qiagen. The extracted DNA was subjected to spectrophotometric analysis for quality and yield estimation. DNA was then stored at $-80^{\circ} \mathrm{C}$ until further analysis.

Three variants (SNPs) rs1321196, rs9364564, and rs3798220 in the Lipoprotein(a) (LPA) gene were genotyped in this study. Target regions were amplified from gDNA using the PCR technique. Primers were designed using the Primer-BLAST tool from NCBI.

Genotyping of exonic missense variant rs3798220 (Location chr 6: 160540105, GRCh38.p12)

The technique of allele-specific PCR (AS-PCR) was used for this variant. Primers were designed with the following sequence:

Forward ancestral: CAAGAACAGCCTAGACACTACT

Forward polymorphic: CAAGAACAGCCTAGACACTACC

Common reverse: ACTGATTCTGGGTGGCCGAG

Genotyping of intronic variants rs1321196 (Location chr 6: 160660810, GRCh38.p12) and rs9364564 (Location chr 6: 160578008, GRCh38.p12)

For rs1321196, forward and reverse primer sequences were: GGCAGTATTGGTCCCAGGTT and ACCTTGGAGGCCATTGTTTGA, respectively.

For rs9364564, forward and reverse primer sequences were: CCCTTGCGCATTTCCCTAGA and CTTTAGGAAGGGCACGGTGT, respectively.

The amplicon was subjected to the RFLP technique to determine the presence or absence of the target variant. Restriction endonuclease Hae III was used for both variants. The products were then separated by agarose gel electrophoresis and visualized using a gel documentation system. Table 1 depicts details of primers, restriction enzymes, amplicon size, and size of digestion products described in the experiments.

The amplicon obtained was directly visualized in a gel documentation system after agarose gel electrophoresis. 
Table 1: Genotyping experiment details of variants with primer sequences, restriction enzyme, amplicon, and digestion product sizes (bp)

\begin{tabular}{llllll}
\hline S. no. & rs ID & Primer sequence & $\begin{array}{l}\text { Restriction } \\
\text { enzyme }\end{array}$ & $\begin{array}{l}\text { Size of } \\
\text { amplicon (bp) }\end{array}$ & $\begin{array}{l}\text { Size of digestion } \\
\text { products (bp) }\end{array}$ \\
\hline 1 & rs3798220 & Forward ancestral/wild-CAAGAACAGCCTAGACACTACT & NA & 211 & NA
\end{tabular}

Forward polymorphic/mutant-CAAGAACAGCCTAGACACTACC

Common reverse-ACTGATTCTGGGTGGCCGAG

2 rs1321196

Forward-GGCAGTATTGGTCCCAGGTT

Reverse-ACCTTGGAGGCCATTGTTTGA

3

rs9364564 Forward-CCCTTGCGCATTTCCCTAGA

Reverse-CTTTAGGAAGGGCACGGTGT
Hae III

359

Hae III

661

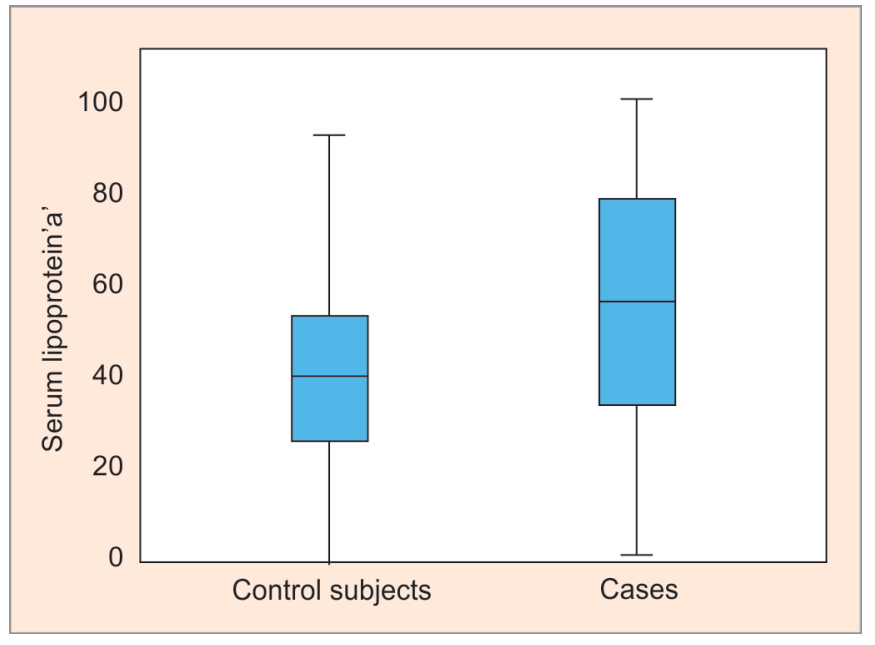

Fig. 2: Comparison of serum lipoprotein (a) between two groups depicted using Box and Whisker plot

the Pearson Chi-square test, which revealed that high levels of serum Lipoprotein(a) is a risk factor for CAD (OR $=3.836 ; 95 \% \mathrm{Cl}=$ $1.735-8.481 ; p$ value $=0.001$ ). Figure 3 shows the bar diagram for distribution of $L p(a)$ using this cutoff, within the study groups. The baseline characteristics of the cases and control are enumerated in Table 2 and the biochemical parameters are listed in Table 3. Traditional coronary risk factors were significantly different between the cases and controls. The lipid parameters were significantly different with levels being significantly lower in cases compared with control owing to the use of cholesterol-lowering medications in this group compared with controls.

\section{Discussion and Clinical Significance}

The mean age of patients suffering from CAD was observed to be 56 years with the majority of patients within the age group of 50-59 years. The mean BMI was 23.55 in the case group, comparable to 23.25 in the control group, both fitting within the normal range of $\mathrm{BMI}$ according to the $\mathrm{WHO}$ criteria. These findings were disquieting and were comparable to the mean BMI observed by Burman et al. 


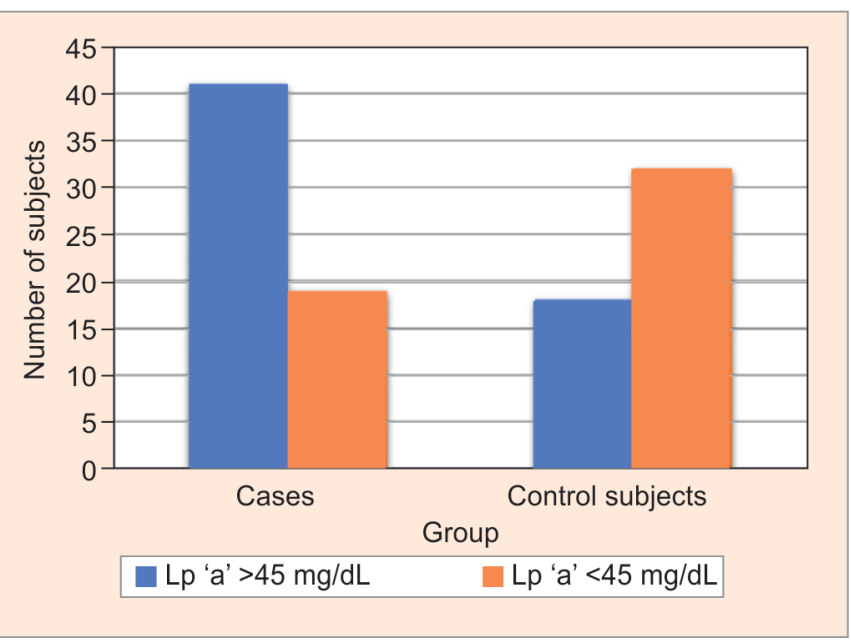

Fig. 3: Distribution of lipoprotein (a) levels within groups along with cutoff decided by ROC analysis

Table 2: Comparison of baseline characteristics between groups

\begin{tabular}{llll}
\hline & $\begin{array}{l}\text { CASE Mean or } \\
\text { percentage of } \\
\text { subjects }\end{array}$ & $\begin{array}{l}\text { CONTROL Mean } \\
\text { or percentage of } \\
\text { subjects }\end{array}$ & p value \\
\hline Parameter & 56.13 & 52.94 & 0.07 \\
\hline $\begin{array}{l}\text { Age (Years) } \\
\text { Gender ratio }\end{array}$ & $54: 6$ & $43: 7$ & 0.518 \\
$(\mathrm{M}: \mathrm{F})$ & & & \\
$\mathrm{BMI}\left(\mathrm{kg} / \mathrm{m}^{2}\right)$ & 23.55 & 23.25 & 0.695 \\
Hypertension & 21.7 & - & - \\
$\begin{array}{l}\text { History of } \mathrm{MI} \text { in } \\
\text { the past }\end{array}$ & 20 & - & - \\
$\begin{array}{l}\text { History of } \\
\text { smoking }\end{array}$ & 16.7 & - & - \\
History of & 3.3 & - & - \\
tobacco use & & - & - \\
$\begin{array}{l}\text { History of } \\
\text { alcohol intake }\end{array}$ & 10 & - & \\
\hline
\end{tabular}

in a North Indian study comprised of patients with CAD. ${ }^{19}$ Hence, we may highlight the finding that, despite having a normal BMI, these patients have $C A D$ with triple vessel disease. Blood for fasting lipid profile was drawn from patients admitted for elective CABG surgery, and hence there was no history of recent myocardial infarction. Serum total cholesterol levels were significantly less with a mean value of $124 \mathrm{mg} / \mathrm{dL}$ in patients compared with 181 $\mathrm{mg} / \mathrm{dL}$ in controls, as patients were on high dose statin therapy (40-80 mg HS). Serum LDL-C levels were low in patients, mean value of $68 \mathrm{mg} / \mathrm{dL}$ compared with $113 \mathrm{mg} / \mathrm{dL}$ in controls. Serum HDL-C was significantly lower in patients, mean value of $34 \mathrm{mg} /$ $\mathrm{dL}$ when compared with the control group $(44 \mathrm{mg} / \mathrm{dL}$ ). These findings are taken together to project the risk posed to the body when it is faced with an amalgamation of unacceptably low levels of protective lipoprotein $\mathrm{HDL}$ and lower than optimal levels of LDL ( $<100 \mathrm{mg} / \mathrm{dL}$ as per NCEP ATPIII guidelines), which may affect the normal functioning of the immune and nervous system from a biochemical viewpoint. ${ }^{20,21}$ Serum alkaline phosphatase was found to be significantly higher in the patient group ( $p$ value $<0.003$ ) dedicating to the view that it can serve as a potential biomarker for the disease. Serum Lipoprotein(a) levels were raised in both patients (mean value $57.49 \pm 3.67 \mathrm{mg} / \mathrm{dL}$ ) and healthy controls
Table 3: Comparison of biochemical parameters between the groups

\begin{tabular}{lccl}
\hline Parameter & Case mean & Control mean & p value \\
\hline $\begin{array}{l}\text { Serum triglycerides } \\
\text { (mg/dL) }\end{array}$ & 133 & 144 & 0.389 \\
$\begin{array}{l}\text { Serum total cholesterol } \\
\text { (mg/dL) }\end{array}$ & 124 & 181 & $0.000^{*}$ \\
HDL-C (mg/dL) & 34 & 44 & $0.000^{*}$ \\
LDL-C (mg/dL) & 68 & 113 & $0.000^{*}$ \\
Total bilirubin (mg/dL) & 0.6 & 0.6 & 0.475 \\
AST (U/L) & 29 & 26 & 0.096 \\
ALT (U/L) & 30 & 26 & 0.335 \\
ALP (U/L) & 90 & 75 & 0.003 \\
Blood urea (mg/dL) & 28 & 25 & 0.584 \\
Serum creatinine $(\mathrm{mg} / \mathrm{dL})$ & 0.7 & 0.8 & 0.327 \\
\hline
\end{tabular}

${ }^{*} p$ values $<0.001$

(mean value $41.68 \pm 3.34 \mathrm{mg} / \mathrm{dL}$ ) with significantly higher mean values in patients ( $p$ value $<0.002$ ) probably leading to CAD. These results were comparable to the findings of studies by Vandana et al., Burman et al., and Ashfaq et al. ${ }^{13,19,22}$ An important finding is that we have observed a significantly higher mean $\mathrm{Lp}(\mathrm{a})$ level $(41.68 \pm 3.34$ $\mathrm{mg} / \mathrm{dL}$ ) in the control group than expected, with a $p$ value $<0.002$. It could be due to the strikingly different genotype and baseline hyperinflammatory state that the Indian population harbors which raise their mean $L p(a)$ levels, yet without causing disease. Another reason to substantiate this finding could be that en-mass measurement of serum $\mathrm{Lp}(\mathrm{a})$ in $\mathrm{mg} / \mathrm{dL}$ is an inferior predictor of the atherogenic potential of the molecule when compared with measurement of the $L p(a)$ particle size. As the number of particles increase, there is a stronger propensity for the development of atherosclerosis. This is a direct contributory effect of the difference in the isoform size of apo(a) each individual carries. This fact must be probed into by further large-scale population studies. An isoform insensitive assay may therefore lead to under or over-estimation of the serum values. Despite the above-mentioned reasons, genetic polymorphisms or epigenetic mechanisms play a sturdy role in the quantity and quality of the molecule generated. A cutoff value of $45 \mathrm{mg} / \mathrm{dL}$ for $\mathrm{Lp}(\mathrm{a})$ was obtained from the ROC curve analysis pointing toward the notion that normal reference range cutoff values must be based on the ethnicity of the population and that we need to re-evaluate our population's baseline values to formulate therapeutic guidelines.

Analysis of SNPs: SNP rs1321196 in Intron 2 caused an increase in serum Lp(a) values in both cases and control subjects. This allele has been observed to increase serum levels of $L p(a)$ probably through a mechanism that involves the binding of transcription factors (YY1, NR4A2) and increased gene transcription. A study by Deo et al. involving 4,464 patients observed a significant influence of the polymorphic allele over serum Lp(a) levels. ${ }^{23}$ However, we noted that the presence of the variant was not limited to patients from our population, as opposed to literature. rs9364564, another less studied intronic variant did not raise serum $L p(a)$ values in our population. In the Third National Health and Nutrition Examination Survey (NHANES III), this polymorphism revealed significant influence in raising serum $L p(a)$ levels in two subpopulations: nonHispanic whites and non-Hispanic blacks but did not show a similar influence on Mexican Americans ${ }^{16}$ akin to our observation. This may be because SNPs of the LPA gene are not solely responsible for the development of disease. Other factors like variation in the 
number of copies of the Kringle IV type II domain influences the major portion of the quantity of $L p(a)$ in serum.

The exonic variant rs3798220, commonly known as the "LPA aspirin genotype" surprisingly did not cause variation in serum $L p(a)$ levels in our population. It is a $T$ to $C$ polymorphism in the apparently inactive protease domain of apo(a), causing isoleucine to be substituted by methionine. This polymorphism is one of the vastly studied missense variants that raise $L p(a)$ levels, from within the LPA gene, across the world. ${ }^{4,24,25}$ Most studies have observed that the presence of the polymorphic allele $C$ increases the risk of CAD twofold. This variant is of clinical interest because carriers of this allele have been shown to benefit from low-dose aspirin therapy as this drug is thought to affect gene transcription. ${ }^{26}$ Hence, this variant carries pharmacogenetic importance to CAD patients worldwide. Unlike as in Western literature, unexpectedly, we found that the presence of the polymorphic allele did not lead to a significant increase in serum $L p(a)$ levels; mean $L p(a)$ levels for genotypes TT (homozygous ancestral), СT (heterozygous), and CC (homozygous polymorphic) being $50.13 \pm 3.42,50.45 \pm 4.17$, and $55 \mathrm{mg} / \mathrm{dL}$, respectively. There was no statistically significant difference between the cases and control group when tallied for the presence of the variant ( $p$ value $<0.590$ ). This fact has been corroborated in a pilot study by Khalifa et al. using samples of patients with CAD from India where they found that this variant did not increase serum $L p(a)$ levels and was not associated with CNVs. ${ }^{27}$

This is an important finding influencing pharmacogenomics and genetic epidemiology of India as we may conclude that Indians may not be affected by this variant even in its presence as the polymorphic allele was distributed evenly between cases and the control subjects. This opens further questions with regards to the exact molecular or epigenetic mechanisms underlying the variation, as proposed transcription factor binding and oxidation of methionine residue in the protein leading to altered binding of apo(a) to extracellular matrix proteins are not sufficient to understand the role of the variant. The observed minor allele frequencies of all variants were significantly higher when compared with the population database of other countries. However, large population-based genome-wide association studies (GWAS) must be conducted to strengthen our findings. The exact role of SNP markers can be elucidated rightly only by large sample size. This study primarily targeted an analytical search for the genetic markers of hyperlipoprotein(a)emia within the LPA gene and their association with the occurrence of CAD. Our study poses few limitations such as restricted sample size, the inclusion of only three variants from the LPA gene, and en-mass measurement of serum $L p(a)$ instead of particle size assessment. However, these factors were outside the scope of the present study.

\section{Conclusion}

Preventive measures and risk stratification of individuals with a predisposition to the development of CAD must be strengthened as the mean age of patients is decreasing over time and non-traditional markers are surfacing strongly against a backdrop of traditional risk factors in India. Forty-eight percent of male and 100\% of female patients fall under the category of premature CAD in our study. We found that serum $\mathrm{Lp}(\mathrm{a})$ levels were not affected by any other variable in the study. Hence, it has been validated as an independent risk factor for the development of CAD. We propose a cutoff value of $45 \mathrm{mg} / \mathrm{dL}$ for $\mathrm{Lp}(\mathrm{a})$ for primary prevention strategies in Indians, after corroboration from larger cohorts in India. High serum Lp(a) levels, though proved to be a causative factor for the development of $C A D$, a uniform isoform insensitive assay needs to be introduced for measurement of the analyte across the country and preferably measured as $L p(a)$ particle number. It is striking to note that the LPA aspirin genotype is not prevalent in our population, which warrants GWAS for CAD specific for India. Furthermore, treatment protocols influenced by the presence of genetic markers may be undertaken only after the creation of a population-specific database as the Indian genotype is heterogeneous and unique.

\section{References}

1. Krishnan MN. Coronary heart disease and risk factors in India - On the brink of an epidemic? Indian Heart J 2012;64(4):364-367. DOI: 10.1016/j.ihj.2012.07.001.

2. India State-Level Disease Burden Initiative CVD Collaborators. The changing patterns of cardiovascular diseases and their risk factors in the states of India: the global burden of disease study 1990-2016. Lancet Glob Health 2018;6(12):e1339-e1351. DOI: 10.1016/S2214109X(18)30407-8.

3. Abdul-Aziz AA, Desikan P, Prabhakaran D, et al. Tackling the burden of cardiovascular diseases in India. Circ Cardiovasc Qual Outcomes 2019;12(4):e005195. DOI: 10.1161/CIRCOUTCOMES.118.005195.

4. Clarke R, Peden JF, Hopewell JC, et al. Genetic variants associated with Lp(a) lipoprotein level and coronary disease. N Engl J Med 2009;361(26):2518-2528. DOI: 10.1056/NEJMoa0902604.

5. Jayasinghe R, Craig IH, Mohan RK. Lipoprotein (A) in clinical practice. J Pak Med Assoc 2014;64(4):447-450.

6. Zlatohlávek L, Zídková K, Vrablík M, et al. Lipoprotein (a) and its position among other risk factors of atherosclerosis. Physiol Res 2008;57(5):777-783. DOI: 10.33549/physiolres.931133.

7. Enas EA, Chacko V, Senthilkumar A, et al. Elevated lipoprotein(a)-a genetic risk factor for premature vascular disease in people with and without standard risk factors: a review. Dis Mon 2006;52(1):5-50. DOI: 10.1016/j.disamonth.2006.01.002.

8. Gabel BR, Koschinsky ML. Sequences within apolipoprotein(a) kringle IV types 6-8 bind directly to low-density lipoprotein and mediate noncovalent association of apolipoprotein(a) with apolipoprotein B-100. Biochemistry 1998;37(21):7892-7898.

9. Gabel BR, Koschinsky MI. Analysis of the proteolytic activity of a recombinant form of apolipoprotein(a). Biochemistry 1995;34(48):15777-15784. DOI: 10.1021/bi00048a023.

10. Schmidt K, Noureen A, Kronenberg F, et al. Structure, function, and genetics of lipoprotein (a). J Lipid Res 2016;57(8):1339-1359. DOI: 10.1194/jlr.R067314.

11. Scanu AM. Lp(a) lipoprotein--coping with heterogeneity. N Engl J Med 2003;349(22):2089-2090. DOI: 10.1056/NEJMp038128.

12. Hoek YY, Wittekoek ME, Wittekoek ME, et al. The apolipoprotein(a) kringle IV repeats which differ from the major repeat kringle are present in variably-sized isoforms. Hum Mol Genet 1993;2(4):361-366. DOI: $10.1093 / \mathrm{hmg} / 2.4 .361$.

13. Ashfaq F, Goel P, Ali W, et al. Lipoprotein (a) levels in relation to severity of coronary artery disease in North Indian patients. Heart Views 2013;14(1):12-16. DOI: 10.4103/1995-705X.107114.

14. Frank S, Klisak I, Sparkes R, et al. The apolipoprotein(a) gene resides on human chromosome 6 q26-27, in close proximity to the homologous gene for plasminogen. Hum Genet 1988;79(4):352-356. DOI: 10.1007/ BF00282175.

15. Maranhão RC, Carvalho PO, Strunz CC, et al. Lipoprotein (a): structure, pathophysiology and clinical implications. Arq Bras Cardiol 2014;103(1):76-84. DOI: 10.5935/abc.20140101.

16. Dumitrescu L, Glenn K, Brown-Gentry K, et al. Variation in LPA is associated with Lp(a) levels in three populations from the third national health and nutrition examination survey. PLoS One 2011;6(1):e16604. DOI: 10.1371/journal.pone.0016604. 
17. Barlera S, Specchia C, Farrall M, et al. Multiple QTL influence the serum $L p(a)$ concentration: a genome-wide linkage screen in the PROCARDIS study. Eur J Hum Genet 2007;15(2):221-227. DOI: 10.1038/ sj.ejhg.5201732.

18. Broeckel U, Hengstenberg C, Mayer B, et al. A comprehensive linkage analysis for myocardial infarction and its related risk factors. Nat Genet 2002;30(2):210-214. DOI: 10.1038/ng827.

19. Burman A, Jain K, Gulati R, et al. Lipoprotein(a) as a marker of coronary artery disease and its association with dietary fat. J Assoc Physicians India 2004;52:99-102.

20. Perez-Guzman C, Vargas MH. Hypocholesterolemia: a major risk factor for developing pulmonary tuberculosis? Med Hypotheses 2006;66(6):1227-1230. DOI: 10.1016/j.mehy.2005.12.041.

21. Moutzouri E, Elisaf M, Liberopoulos E. Hypocholesterolemia. Curr Vasc Pharmacol 2011;9(2):200-212. DOI: 10.2174/15701611 1794519354.

22. Vandana S, Bhatnagar MK, Jayashree B. Evaluation of homocysteine, lipoprotein(a) and endothelin as diagnostic markers of coronary artery disease in Indian population. Internet J Med Update 2013;8(1):17-23.
23. Deo RC, Wilson JG, Xing C, et al. Single-nucleotide polymorphisms in LPA explain most of the ancestry-specific variation in Lp(a) levels in African Americans. PLoS One 2011;6(1):e14581. DOI: 10.1371/journal. pone.0014581.

24. Arai K, Luke MM, Koschinsky ML, et al. The $14399 \mathrm{M}$ variant of apolipoprotein(a) is associated with increased oxidized phospholipids on apolipoprotein B-100 particles. Atherosclerosis 2010;209(2):498-503. DOI: 10.1016/j.atherosclerosis.2009. 09.077.

25. Shiffman D, Kane JP, Louie JZ, et al. Analysis of 17,576 potentially functional SNPs in three case-control studies of myocardial infarction. PLoS One 2008;3(8):e2895. DOI: 10.1371/journal.pone. 0002895.

26. Chasman DI, Shiffman D, Zee RY, et al. Polymorphism in the apolipoprotein(a) gene, plasma lipoprotein(a), cardiovascular disease, and low-dose aspirin therapy. Atherosclerosis 2009;203(2):371-376.

27. Khalifa M, Noureen $A$, Ertelthalner $K$, et al. Lack of association of rs3798220 with small apolipoprotein(a) isoforms and high lipoprotein(a) levels in East and Southeast Asians. Atherosclerosis 2015;242(2):521-528. 


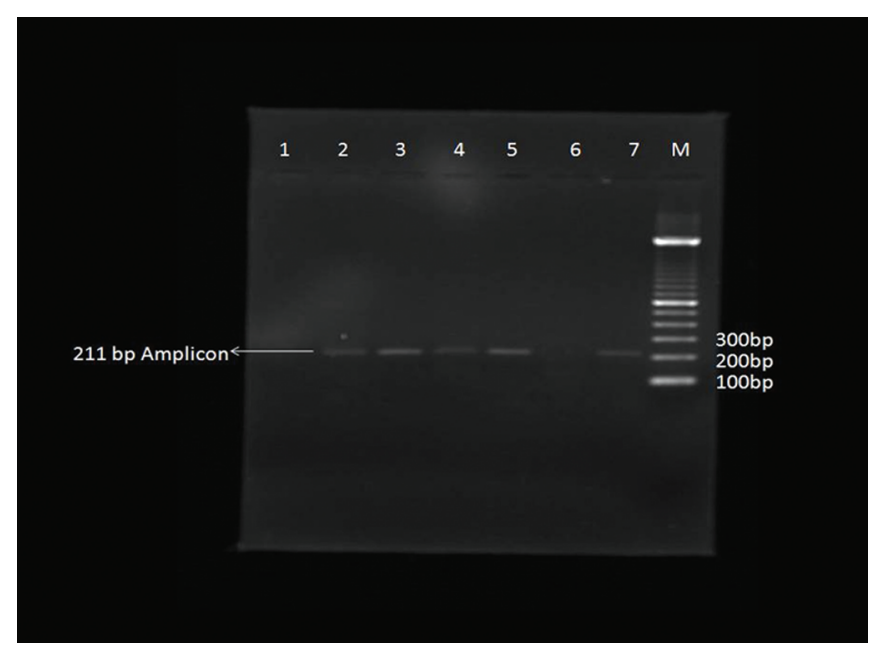

Supplementary Fig. 1: Agarose gel electrophoresis for rs3798220; M: molecular weight marker, Lane 2 and 3, 4 and 5: heterozygous (TC), Lane 6 and 7: homozygous ancestral (TT)

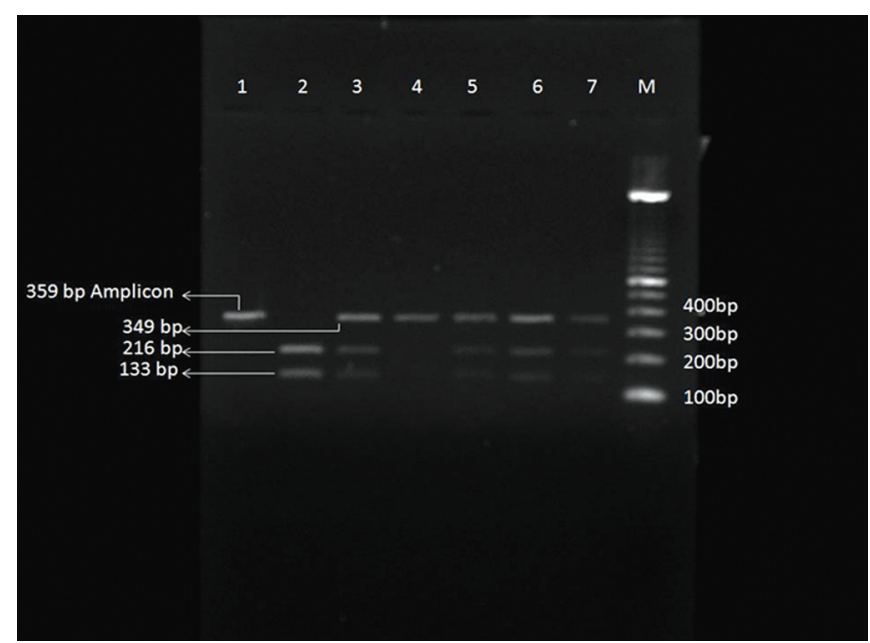

Supplementary Fig. 3: Agarose gel electrophoresis for rs1321196; M molecular weight marker, Lane 2: homozygous ancestral (GG), Lanes $3,5,6,7$ : heterozygous (GA), Lane 4: homozygous polymorphic (AA)

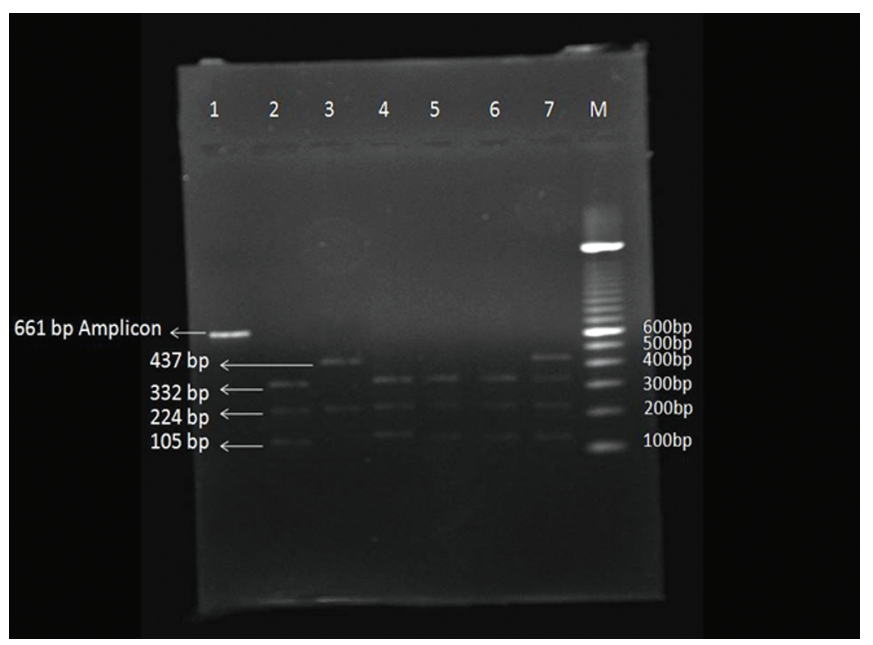

Supplementary Fig. 2: Agarose gel electrophoresis for rs9364564; M: molecular weight marker, Lane 2, 4, 5, 6: homozygous ancestral (GG), Lane 3: homozygous polymorphic (AA), Lane 7: heterozygous (GA)

Supplementary Table 1: SNP variant rs3798220: the size of amplicon and details of AS-PCR products

\begin{tabular}{ll}
\hline Size of amplicon (bp) & Products visualized \\
\hline 211 & TT: Product amplified only in the tube with \\
& forward ancestral primer and reverse primer \\
& CC: Product amplified only in the tube with \\
& forward polymorphic primer and reverse \\
& primer \\
& TC: Product amplified in both tubes \\
\hline
\end{tabular}

Supplementary Table 2: SNP variant rs9364564: the size of amplicon and Hae III digested products

\begin{tabular}{ll}
\hline & rs9364564 \\
\hline Size of amplicon $(b p)$ & Size of digested products $(b p)$ \\
\hline 661 & GG: $332-224-105$ \\
& AA: 437-224 \\
& GA: 437-332-224-105 \\
\hline
\end{tabular}

Supplementary Table 3: SNP variant rs1321196: the size of amplicon and Hae III digested products

\begin{tabular}{ll}
\hline & rs1321196 \\
\hline Size of amplicon $(b p)$ & Size of digested products $(b p)$ \\
\hline 359 & GG: $216-133-10$ \\
& AA: $349-10$ \\
& GA: $349-216-133-10$ \\
\hline
\end{tabular}

